\title{
Crónica de un cambio anunciado. Las elecciones de 2017 en Chile frente al cambio de sistema electoral
}

\author{
Facundo Cruz (Argentina)* \\ Carlos Varetto (Argentina) ${ }^{* *}$
}

\section{Resumen}

Este artículo se enfoca en el análisis de las elecciones presidenciales en Chile de 2017 en perspectiva comparada desde el retorno a la democracia en 1989, en el que se sostiene que el sistema partidario se encuentra en una nueva etapa de su trayectoria que comienza en 2013 y en la que ambos sistemas partidarios presidencial y legislativo- comienzan a confluir nuevamente en lo que respecta a sus niveles de fragmentación y volatilidad electoral. Es posible que este nuevo formato y una nueva dinámica competitiva tiendan a consolidarse. De esta manera, se estaría dando lugar a una tercera etapa. La primera del sistema partidario chileno tuvo lugar entre 1989 y 2005 caracterizada por la estabilidad, baja fragmentación y alta institucionalización. La segunda transcurrió entre 2005 y 2013, en la que se destaca un desajuste entre competencia presidencial — progresivamente más volátil y fragmentada- y legislativa — aún estable y menos fragmentada-.

\section{Palabras clave}

Sistema Electoral; Elecciones; Cambio Político; Sistema de Partidos; Chile.

Fecha de recepción: agosto de 2018

- $\quad$ Fecha de aprobación: octubre de 2018

\section{Cómo citar este artículo}

Cruz, Facundo y Varetto, Carlos. (2019). Crónica de un cambio anunciado. Las elecciones de 2017 en Chile frente al cambio de sistema electoral. Estudios Políticos (Universidad de Antioquia), 54, pp. 233-258. http://doi.org/10.17533/ udea.espo.n54a12

\footnotetext{
* Licenciado en Gobierno y Relaciones Internacionales. Magíster en Análisis, Derecho y Gestión Electoral. Doctor en Ciencia Política. Profesor investigador Instituto de Investigación «Gino Germani», Universidad de Buenos Aires (UBA), Argentina. Correo electrónico: cruzfacu@gmail.com

${ }^{* *}$ Licenciado en Sociología. Doctor en Ciencia Política. Investigador Asistente del Consejo Nacional de Investigaciones Científicas y Tecnológicas (Conicet) y profesor regular de la Universidad Nacional de San Martín (Unsam). Correo electrónico: cvaretto@unsam.edu.ar
} 


\title{
Chronicle of an Announced Change. The 2017 Chile's Elections Facing the Change in the Electoral System
}

\begin{abstract}
This article analyses the 2017 presidential elections in Chile based on a comparative perspective that takes into account the period beginning with the return to democracy in 1989 . We maintain that the party system is in a new stage of its trajectory that started in 2013 and in which both party systems (presidential and legislative) began to converge once again in terms of their levels of electoral fragmentation and volatility. The context shows the possibility of the consolidation of this new format and a new competitive dynamic. In this way, it would be leading to a third stage. The first stage of the Chilean party system took place between 1989 and 2005 characterized by stability, low fragmentation and high institutionalization. A second stage took place between 2005 and 2013, in which we highlight a mismatch between the presidential competition (progressively more volatile and fragmented) and the legislative competition (still stable and less fragmented).
\end{abstract}

Keywords

Electoral System; Elections; Political Change; Party System; Chile. 
Crónica de un cambio anunciado. Las elecciones de 2017 en Chile frente al cambio...

\section{Introducción}

El presente artículo tiene por objeto analizar las elecciones generales de 2017 en Chile en perspectiva diacrónica con los distintos procesos electorales que se celebraron desde el retorno a la democracia en 1989. En particular, se propone insertar los resultados electorales en el marco de las principales tendencias del sistema partidario y su estructura de competencia desde el regreso a la democracia y evaluar los futuros escenarios que puedan surgir a raíz de la modificación del sistema electoral para diputados y senadores nacionales.

Existe un consenso generalizado en destacar al sistema partidario chileno como estable y altamente institucionalizado (Scully, 1995; Coppedge, 1998; Siavelis, 2000, 2004; Torcal y Mainwaring, 2003; Buquet, 2015). De hecho, los partidos políticos chilenos han sido considerados como interlocutores válidos en la arena política y fuertemente implantados en la sociedad (Cavarozzi y Casullo, 2002), caracterizada por una alta estabilidad de sus votantes (López y Baeza, 2013). La mayoría de estos trabajos, sin embargo, se han concentrado en estudiar los primeros quince años de la democracia partidaria chilena. Nuevos estudios han puesto en duda estos hallazgos, en especial, en lo que refiere a los vínculos de los partidos políticos con la sociedad (Morgan y Meléndez, 2016; Siavelis, 2016) y su posterior impacto en el sistema partidario (Luna y Altman, 2011; Luna y Rosenblatt, 2012). Tal como marcaron David Altman y Juan Pablo Luna (2015, p. 219), el sistema binominal chileno bloqueó, en cierta medida, «una evolución dinámica y una renovación moderada del sistema de partidos». Esto resultó en una estructura de competencia diferenciada entre el sistema partidario presidencial y el legislativo. Ambos subsistemas han interactuado guiados por diferentes reglas, lo que implicó que mientras que para la elección presidencial se reflejaron nuevas tendencias, el ámbito parlamentario se mantuvo inalterado. ${ }^{1}$ Lo que la competencia presidencial venía anticipando en elecciones anteriores se terminó de manifestar en la dimensión legislativa en las últimas elecciones generales, favorecido por la modificación de las reglas electorales.

Las elecciones de 2017 representan el punto de confluencia del sistema partidario presidencial y del legislativo en Chile, lo que a futuro, tal vez, pueda consolidarse como un nuevo formato y una nueva dinámica competitiva. Para

\footnotetext{
${ }^{1}$ Resulta adecuado comprender al sistema partidario como un conjunto de subsistemas donde los actores pueden realizar potencialmente estrategias diferentes, incluso en el caso bajo análisis, donde las reglas y estímulos institucionales son claramente diferentes (Bardi y Mair, 2008).
} 
indagar en estas cuestiones —y en coincidencia con Tomas Došek (2016)— se distingue entre tres etapas del sistema partidario: la primera se desarrolla entre 1989 y 2005; la segunda entre 2005 y 2013; y la tercera comienza en 2013 y se extiende hasta las últimas elecciones celebradas — aún sin rumbo cierto-.

\section{El contexto previo a las elecciones}

Hay algunas cuestiones centrales para comprender el proceso político antecedente a las elecciones presidenciales de 2017 en Chile. Entre ellas se destacan el proceso de desgaste político y económico del gobierno, la fragmentación de la oferta partidaria, en especial del espectro de izquierda las fuerzas habitualmente coalicionadas en Concertación o Nueva Mayoría-, y la aplicación por primera vez de la reforma en el ámbito legislativo que terminó con el sistema binominal.

En lo que refiere a la situación del gobierno, las señales desde la economía para sustentar las aspiraciones electorales eran poco alentadoras.

Gráfica 1. Variación interanual producto interno bruto en Chile, 2013-2017 (\%).

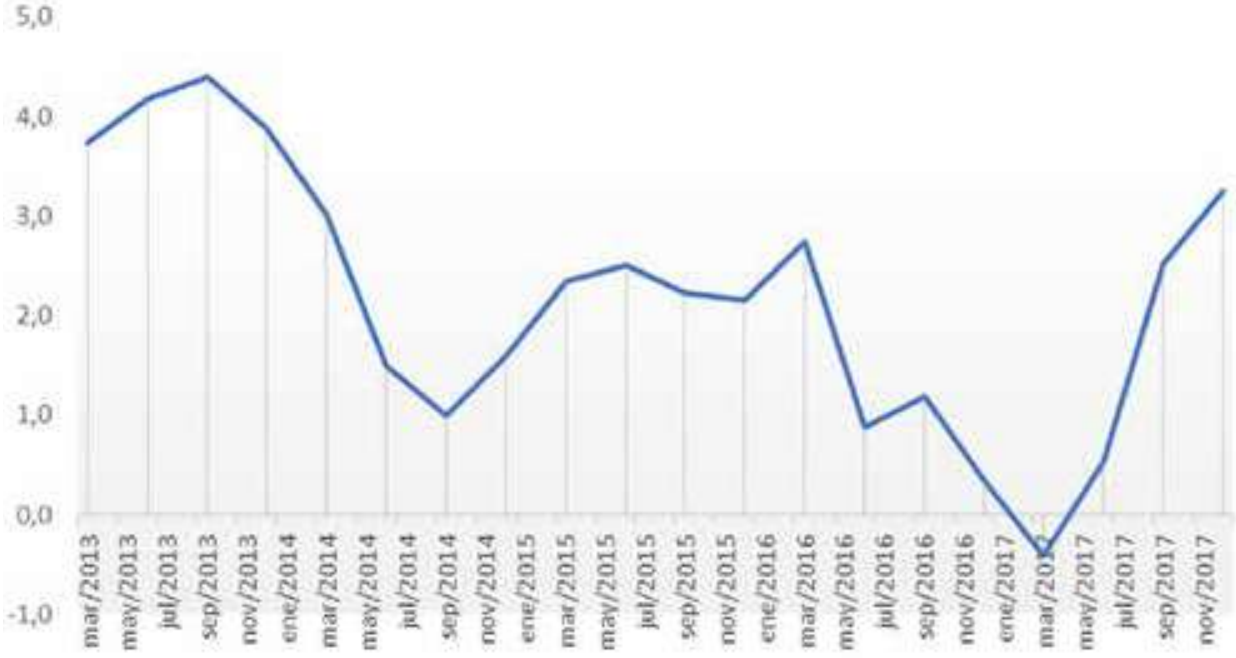

Fuente: Banco Central de Chile.

Desde junio de 2016 el crecimiento del PIB se encontraba en desaceleración a tal punto que, en marzo de 2017, apenas unos meses antes de las elecciones primarias presidenciales, tuvo un rendimiento negativo. 
No obstante, alcanzó una mejoría antes de la primera ronda electoral. Estas dificultades en el frente económico estuvieron acompañadas por valoraciones negativas de la opinión pública sobre la gestión presidencial y la situación del país. Apenas 18\% aprobaba la gestión del Gobierno en mayo de 2017: el porcentaje más bajo desde el año 2000 y 30 puntos por debajo de sus primeros meses en el segundo gobierno. En tanto que solo $17 \%$ afirmaba que el país estaba progresando (Centro de Estudios Públicos, s. f.).

Este deterioro pudo estar vinculado al magro rendimiento económico del país, pero también se vio enfrentado al desgaste por las iniciativas de reforma del gobierno ${ }^{2} y$ a algunas de las crisis institucionales que tuvieron lugar durante 2017. Específicamente, Sergio Toro Maureira y Macarena Valenzuela (2018) marcan las tensiones de Bachelet con su gabinete económico que en agosto de 2017 llevaron a la renuncia a dos ministros y un subsecretario, y dos eventos críticos de significativa envergadura: la crisis de Carabineros por desfalco de fondos y el abuso y muerte de niños y niñas bajo tutela del Estado en los centros del Servicio Nacional de Menores (Sename).

En este marco de relativa debilidad del Gobierno, no resulta llamativo que se le haya dificultado sostener la integridad de la coalición, incrementando la fragmentación en el espacio de centroizquierda. En el ámbito formal de la coalición, se destacó el desprendimiento de la Democracia Cristiana (DC) por primera vez desde el regreso a la democracia. Este alejamiento del oficialismo estuvo vinculado a diferencias sobre el diagnóstico respecto al camino a seguir respecto el sistema tributario y los derechos laborales, así como por diferentes consideraciones sobre el derecho al aborto, antes que por las reformas llevadas adelante por el gobierno de Bachelet (Seira et al., 2017). Así, la DC apuntó a volver a constituirse como una alternativa de centro.

Por otro lado, en enero de 2017 se lanzó el Frente Amplio, en el que algunos de sus integrantes eran disidentes de la Nueva Mayoría. Tal es el caso de Revolución Democrática, la cual se gestó al calor de las movilizaciones estudiantiles de 2011 y que mantuvo - aun con disidencias- cercanía con la Nueva Mayoría e incorporó cuadros en el Ministerio de Educación durante el gobierno de Bachelet, los cuales se alejaron definitivamente en 2016 ( $E m o l$, 2016, mayo 23). A esta oferta se sumó la candidatura de Marco EnríquezOminami, quien se desvinculó de la Concertación para lanzar su candidatura

${ }^{2}$ Para una revisión del primer impulso de las reformas del gobierno de Bachelet, véase David Altman y Sergio Toro Maureira (2016). 
presidencial en 2009. Finalmente, dos candidaturas con menores respaldos se sumaron a la oferta de izquierda: PAIS, escindido del Frente Amplio, y Unión Patriótica (UPA). En cambio, las fuerzas de centro-derecha lograron sostenerse relativamente indemnes y dirimir la candidatura en elecciones primarias salvo por la salida de José Antonio Kast- para competir como independiente en la elección presidencial.

Por último, resulta central la puesta en práctica en 2017 de un nuevo sistema electoral en el ámbito legislativo, que dejó atrás ese experimento único que fue el sistema binominal para la elección de congresistas. El sistema se caracterizaba por una magnitud igual a dos en cada distrito. Para que un partido o coalición se pudiera alzar con ambas bancas debía doblar en votos a la segunda lista. En caso de no hacerlo, cada uno se llevaba una banca. En la práctica, no tuvo un efecto reductor significativo sobre las candidaturas, pero sí sobre la cantidad de fuerzas que obtuvieron escaños desde 1989. Esto se vio reforzado por la simultaneidad con la elección presidencial y por la tendencia a la búsqueda de la reelección de los congresistas (Cabezas y Navia, 2005). La nueva ley, sancionada en 2015, estableció el sistema de asignación de bancas proporcionales con base en el método D’Hondt, con veintiocho distritos y quince circunscripciones de magnitudes medianas y variables según la población. A la vez que instituyó el aumento de parlamentarios, la disminución de barreras para la creación de partidos políticos y mayor participación femenina. En este último punto, se estableció que ni los candidatos hombres ni las candidatas mujeres pueden superar el $60 \%$, a la vez que se fijaron medidas de fomento por candidatas electas por cada fuerza, como también reembolsos de gastos para candidatas electas (Servel, 2015, agosto 18).

En lo que se refiere a la categoría presidencial no hubo cambios. La elección de presidente en Chile es directa, a distrito único y con sistema de mayoría absoluta. Caso contrario, se realiza una segunda vuelta entre los dos candidatos más votados, con cinco semanas de diferencia. La primera vuelta presidencial se realizó en simultáneo con la de diputados, senadores y consejeros regionales. El cargo de presidente posee un mandato de cuatro años, no habilita la reelección inmediata, ni hay cargo de vicepresidente en juego. ${ }^{3}$

\footnotetext{
${ }^{3}$ Cabe agregar que entró en vigor, desde 2013, la Ley 20568 que establece el sistema de voto voluntario y de inscripción automática para las elecciones. En lo que refiere a una revisión de la evolución del sistema electoral y sus componentes hasta 2015, véase Došek (2016).
} 
En el ámbito ejecutivo se utilizó por segunda vez el sistema de primarias sancionado en 2012, el cual establece la obligación de realizar elecciones internas solo en aquellas que presenten un número superior de candidatos que los cargos a definir. Por otro lado, no todos los electores participan de la elección, sino solo los afiliados de los pactos electorales participantes o independientes habilitados, quienes tendrán que elegir de cuál primaria ser parte, ya que no podrán votar más de una vez. En el caso de los militantes de los pactos o partidos que no participan en el proceso no están habilitados para sufragar en las primarias, habilitados para hacerlo en las elecciones generales. También se aplicó por primera vez la posibilidad de que los ciudadanos chilenos con derecho a sufragio que se encuentren fuera de Chile emitan su voto, la cual aplica únicamente a la elección de presidente y las posibles primarias presidenciales (Servel, s. f. a).

\section{Los candidatos y sus programas}

Como se indicó más arriba, las elecciones presidenciales contemplan la posibilidad de elecciones primarias para aquellos partidos con múltiples candidatos y una segunda vuelta electoral entre las dos opciones más votadas si ninguna supera el 50\% de los votos. En este marco, en 2017 las elecciones primarias tuvieron lugar el 2 de julio, la primera ronda electoral el 19 de noviembre y la segunda el 17 de diciembre.

En las elecciones primarias participaron únicamente Chile Vamos y el Frente Amplio. Si bien hasta abril de 2017 Nueva Mayoría —coalición gobernante- tenía proyectado realizar su primaria presidencial en conjunto con las de los demás pactos, la ruptura con la Democracia Cristiana llevó a que los candidatos de los partidos de la coalición —Alejandro Guillier y Carolina Goic_ - fueran directamente a la primera vuelta de la elección presidencial.

Chile Vamos se presentó integrada por los partidos Unión Demócrata Independiente, Renovación Nacional, Evolución Política (Evopoli) y Partido Regionalista Independiente. Tres precandidatos presidenciales se presentaron en las primarias: el expresidente Sebastián Piñera, su exministro de Desarrollo Social Felipe Kast (Evopoli) y Manuel José Ossandón (independiente). ${ }^{4}$ Piñera logró vencer con 58,36\% contra 15,4 \% de Kast y 26,24\% de Ossandón (Servel, 2017, julio 2).

${ }^{4}$ Se desempeño como alcalde de Puente Alto (2000-2012) y como senador por la circunscripción Santiago Oriente (2014-2017). 
Por su parte, el Frente Amplio estuvo integrada por los partidos Revolución Democrática, Humanista, Liberal, Ecologista Verde, Poder Ciudadano e Igualdad. Esta fuerza dirimió su candidato entre la periodista Beatriz Sánchez, quien contó con el respaldo de Revolución Democrática, la Izquierda Libertaria, el Movimiento Autonomista y el Partido Humanista, y el exdirector del departamento de Sociología de la Facultad de Ciencias Sociales de la Universidad de Chile, Alberto Mayol, con el apoyo del movimiento Nueva Democracia. Beatriz Sánchez obtuvo 67,56\%, contra a 32,44\% de Alberto Mayol.

Definidas las candidaturas presidenciales, el 19 de noviembre siguiente se pusieron en juego la titularidad del Palacio de la Moneda: 23 senadores, 155 diputados y 278 consejeros regionales elegidos en circunscripciones provinciales. Los candidatos principales fueron Alejandro Guiller por la coalición oficialista Nueva Mayoría, el expresidente Sebastián Piñera por Chile Vamos y, un paso más atrás, Beatriz Sánchez del Frente Amplio. En lo que sigue, se da cuenta panorámicamente de los candidatos, sus perfiles y sus posicionamientos programáticos destacados.

El candidato de Nueva Mayoría ${ }^{5}$ fue Alejandro Guillier, identificado con [240] una dilatada trayectoria como periodista. En febrero de 2013 dejó el canal de televisión abierta La Red, para postularse como candidato al Senado de Chile en las elecciones de ese año, con el apoyo del PRSD. El 9 de abril de 2017 logró el respaldo del PS, luego de vencer al candidato del PPD y expresidente de la República, Ricardo Lagos, en una votación realizada por el Comité Central (Cooperativa.cl, 2017, abril 9). Durante la campaña Guiller se mostró como un continuador del camino de reformas recorrido por la gestión de Bachelet, retomó la reforma inconclusa de Bachelet correspondiente a «Educación superior gratuita para el 70 por ciento de los estudiantes más vulnerables», sumándole el fin al Crédito con Aval del Estado (CAE) ${ }^{6}$ y

\footnotetext{
${ }^{5}$ En las elecciones de 2017 se presentó con el nombre de la Fuerza de la Mayoría. Es la coalición heredera de la Concertación, ubicada en la centro-izquierda e integrada por los partidos políticos que empujaron la transición a la democracia a finales de la década de 1980: el Partido Socialista (PS), el Partido por la Democracia (PPD), la Democracia Cristiana (DC) y el Partido Radical-Socialdemócrata (PRSD). Desde las elecciones de 2009 el Partido Comunista (PC) se unió al acuerdo y pasó a llamarse Nueva Mayoría. En 2013 se sumó el Movimiento Amplio Social (MAS). En este artículo se le denomina a este espacio Concertación-Nueva Mayoría.

${ }^{6}$ Beneficio del Estado otorgado a través del sistema financiero a estudiantes de probado mérito académico que necesitan apoyo financiero para iniciar o continuar una carrera en alguna de las instituciones de educación superior (IES) acreditadas que forman parte del Sistema de Crédito con
} 
proponiendo como solución gradual el 1\% de interés real y, si se pierde el trabajo, cancelar el cobro (NTN24, 2017, diciembre 12). También propuso una reforma constituyente para llevar al país hacia el semipresidencialismo (Cooperativa.cl 2017, noviembre 8) y un ambicioso plan de descentralización (Fortín Mapocho, 2017, octubre 23). Finalmente, en su campaña se destacaron la propuesta de intensificar la inversión pública en empleo, especialmente en construcción, y la reforma del sistema de pensiones a través de un método tripartito y mixto en el que el Estado pase de su actual rol subsidiario a uno en el que garantice la calidad de vida y jubilaciones dignas para adultos mayores (Guillier, 2017).

Por su parte, la Democracia Cristiana, por primera vez compitiendo en solitario desde 1970, presentó a Carolina Goić, una política de dilatada trayectoria que ejerció como diputada entre 2006 y 2014, posteriormente como senadora y desde 2016 como presidente del partido. El programa de campaña rescató lo avanzado en Chile, pero dio cuenta de la necesidad de un nuevo liderazgo con mirada de largo plazo, que evite caminos populistas y simplistas, para superar la «trampa del ingreso medio» y el «desarrollo frustrado» (Goić, 2017). Su propuesta hizo hincapié en recuperar el crecimiento económico, aunque también se destacaron la propuesta de crear un Ministerio de Seguridad Pública y una reforma a la salud, con énfasis en una nueva ley del cáncer (Guzmán, 2017, octubre 31).

El Frente Amplio presentó como candidata a Beatriz Sánchez, una popular periodista con una trayectoria vinculada a la agenda de género. El discurso de campaña se construyó sobre la idea de una construcción colectiva e igualitarista, denunciando la contracara del elitismo de los demás partidos. En este marco, su programa destacó que «cualquiera pueda llegar a ser presidente de la República». La idea de construcción colectiva se plasmó en un documento que denominaron El programa de muchos, que fue puesto bajo escrutinio a través de una consulta ciudadana (El Desconcierto, 2017, septiembre 27) y que en su versión final contempló una política estatal de nacionalización de empresas, cambios en el Congreso, agenda trinacional con Bolivia y Perú, asamblea constituyente en busca del establecimiento de un Estado plurinacional e intercultural, despenalización del aborto sin causales hasta el primer trimestre, el fin de las administradoras de fondos de pensiones (AFP) y educación gratuita (Diario Uchile, 2017, octubre 21).

Garantía Estatal. Las exigencias mínimas están reglamentadas por la Ley 20027 y su reglamento, y por lo que establece cada institución de educación superior (Ingresa, s. f.). 
El Frente Amplio también tuvo su fractura, con la salida de PAIS y la presentación como candidato presidencial de Alejandro Navarro, senador y de militancia previa en el Partido Socialista. Otro candidato dentro del espectro de izquierda fue Marco Enríquez-Ominami, ex dirigente socialista y diputado por ese partido durante dos periodos. No fue su debut, ya había competido por la Presidencia en 2009 por fuera de la Concertación como independiente y en 2013 con su Partido Progresista (PRO). En las elecciones de 2017 volvió a presentarse con PRO. En un posicionamiento similar a Guillier, propuso sostener la agenda y trabajar por mejorar la economía (Sputnik, 2017, octubre 19). La lista de candidatos de orientación de izquierda se completó con Eduardo Artés de Unión Patriótica (UPA), un histórico líder del Partido Comunista Chileno (Acción Proletaria), y el ya mencionado Alejandro Navarro por PAIS. Ninguno de los dos alcanzó siquiera el 1\% de los votos.

Chile Vamos es la coalición sucesora de Alianza por Chile, ${ }^{7}$ fundada durante el retorno a la democracia en 1989 por los partidos Unión Demócrata Independiente (UDI) y Renovación Nacional (RN). En 2017 incorporó al Partido Regionalista Independiente (PRI) y al nuevo partido liberal, Evolución Política (Evópoli). El eje de la campaña de Piñera se enfocó en vincular las reformas llevadas adelante por el gobierno encabezado por Bachelet con la desaceleración del crecimiento económico. El programa de gobierno destacó: «estamos perdiendo el rumbo y el ritmo porque el gobierno de la nueva mayoría nos está conduciendo por el camino equivocado. A partir de un diagnóstico erróneo y una ideología equivocada, ha puesto en marcha un conjunto de iniciativas mal pensadas y peor implementadas» (Piñera, 2017). Dentro de sus propuestas de campaña resaltaron las siguientes: modernizar la legislación en materia de jornada laboral; la creación de un nuevo sistema solidario de acceso a la educación superior, el cual garantizara becas, créditos estatales y la gratuidad en algunos casos académicos; la modernización y el fortalecimiento de Carabineros de Chile y de la Policía de Investigaciones a través de reformas administrativas y operativas; mejorar, de manera gradual, las pensiones y aumentar recursos para Pilar Solidario de Pensiones — beneficio del Estado para aumentar el ahorro provisional de trabajadores- (NTN24, 2017, diciembre 12).

\footnotetext{
${ }^{7}$ Desde 1989 esta coalición electoral de centro-derecha ha ido cambiando su nombre, pero siempre manteniendo su composición interna: Democracia y Progreso (1989), Unión por el Progreso de Chile (1993), Alianza por Chile (1999 y 2005), Coalición por el Cambio (2009), Alianza (2013) y Chile Vamos (2017). Durante esos años partidos menores de la derecha y centro-derecha chilena se aliaron a RN y UDI. En este artículo se refiere a este espacio como Alianza-Chile Vamos.
} 
Por último, la derecha también tuvo su defección por la candidatura independiente de José Antonio Kast, que se presentó tras haber renunciado a la Unión Democrática Independiente. ${ }^{8}$ Aunque fue marginal en términos electorales, tuvo su impacto mediático a raíz de las polémicas posturas públicas que adquirió el candidato en torno a cuestiones como la igualdad de derechos (De la Paz, 2017, diciembre) y el impacto de la dictadura de Pinochet en la sociedad chilena (Montes, 2017, noviembre 13). El programa propuso cuatro ejes centrales: seguridad y Estado de derecho; urgencias sociales; Estado moderno; y reactivación económica y emprendimiento. Sugirió derogar las reformas educativas, laborales y tributarias aprobadas durante el gobierno de Bachelet (Opinión Global, 2017, julio 17).

En síntesis, los chilenos el 19 de noviembre de 2017 se encontraron con una oferta de ocho candidatos presidenciales, de los cuales prevalecieron aquellos que se ubicaron a la centro-izquierda. También se encontraron en la escena a candidatos que podrían considerarse como outsiders: Beatriz Sánchez, sin duda, y Alejandro Guillier, en menor medida.

\section{La competencia presidencial, que ya se venía cantando}

La competencia presidencial de 2017 adquirió algunos componentes tradicionales de la disputa política chilena desde el retorno a la democracia en 1989, pero otros particulares y específicos que muestran un proceso de transición en su sistema partidario. No es posible sostener la existencia de una ruptura con los patrones de competencia partidaria en Chile, pero sí una lenta evolución hacia una dinámica competitiva distinta. Nuevamente, la titularidad del Poder Ejecutivo tuvo que resolverse en la instancia de ballotage. De las siete elecciones presidenciales desde 1989 hasta la fecha, solamente en dos de ellas —1989 y 1993 - fue suficiente una sola vuelta: Patricio Aylwin y Eduardo Frei Ruiz-Tagle — ambos de la DC—. En los cinco restantes se enfrentaron en una segunda vuelta los candidatos de las dos principales coaliciones electorales que han dominado la política chilena en los últimos treinta años: la Concertación-Nueva Mayoría y Alianza-Chile Vamos.

Las elecciones presidenciales de 2017 no escaparon a esta dinámica. En la primera vuelta presidencial, Sebastián Piñera —Chile Vamos-RNobtuvo 36,64\% de los votos y Alejandro Guiller — La Fuerza de la MayoríaIndependiente-22,70\%. En un escenario similar al de las elecciones de 2009

${ }^{8}$ Fue un referente partidario, se desempeñó como jefe de bloque de diputados de la UDI desde principios de la década de 2000. 
(Castiglioni, 2010; López y Baeza, 2013), la división de la centro-izquierda chilena en dos coaliciones con candidatos propios dificultó la posibilidad de obtener el primer lugar y llegar al ballotage con más fuerza. El tercer lugar lo ocupó el Frente Amplio con 20,27\%. Este escenario dejó el resultado final en suspenso hasta la segunda vuelta el 17 de diciembre. Piñera resultó electo presidente por segunda vez, al alcanzar $54,58 \%$ de los votos, frente a $45,42 \%$ de Guillier. Otra vez el escenario de ocho años atrás.

En algunos aspectos, este resultado muestra ciertas regularidades que han estado presentes en todas las elecciones presidenciales chilenas desde 1989. De la tabla 1 se desprenden dos cuestiones clave: en primer lugar, que todos los candidatos que concurrieron al ballotage y ganaron la primera vuelta resultaron electos presidentes; en segundo lugar, que los candidatos ganadores de la primera vuelta lo hicieron con comodidad cuando la carrera presidencial contó con más de dos candidatos competitivos — valores número efectivo de partidos presidenciales (NEPP) ${ }^{9}$ cercanos o pasando los 2,5-. En aquellos casos donde el NEPP se acercó a 2 el margen de victoria (MV) entre primero y segundo se redujo drásticamente.

Tabla 1. Margen de victoria (MV) y número efectivo de partidos (NEP) en elecciones presidenciales en Chile, 1989-2013. ${ }^{10}$

\begin{tabular}{|c|c|c|c|c|}
\hline Año & Primer lugar & Segundo lugar & $\begin{array}{c}\text { MV } \\
\text { presidencial }\end{array}$ & $\begin{array}{c}\text { NEP } \\
\text { presidencial }\end{array}$ \\
\hline 1989 & $\begin{array}{c}\mathbf{5 5 , 1 7 \%} \\
\text { (Concertación-DC) }\end{array}$ & $\begin{array}{c}29,40 \% \\
\text { (Democracia y Progreso-UDI) }\end{array}$ & $25,77 \%$ & 2,41 \\
\hline 1993 & $\begin{array}{c}57,98 \% \\
\text { (Concertación-DC) }\end{array}$ & $\begin{array}{c}24,41 \% \\
\text { (Unión por el Progreso-UDI) }\end{array}$ & $33,57 \%$ & 2,47 \\
\hline 1999 & $\begin{array}{c}47,95 \% \\
\text { (Concertación-PS-PPD) }\end{array}$ & $\begin{array}{c}47,51 \% \\
\text { (Alianza-UDI) }\end{array}$ & $0,44 \%$ & 2,19 \\
\hline 2005 & $\begin{array}{c}\mathbf{4 8 , 6 4 \%} \\
\text { (Concertación-PS) }\end{array}$ & $\begin{array}{c}45,96 \% \\
\text { (Alianza-RN) }\end{array}$ & $2,68 \%$ & 2,22 \\
\hline 2009 & $\begin{array}{c}\mathbf{4 4 , 0 6 \%} \\
\text { (Coalición por el Cambio-RN) }\end{array}$ & $\begin{array}{c}\mathbf{2 9 , 6 0 \%} \\
\text { (Concertación-DC) }\end{array}$ & $14,46 \%$ & 3,07 \\
\hline 2013 & $\begin{array}{c}46,67 \% \\
\text { (Nueva Mayoria-PS) }\end{array}$ & $\begin{array}{c}25,01 \% \\
\text { (Alianza-UDI) }\end{array}$ & $21,66 \%$ & 3,29 \\
\hline 2017 & $\begin{array}{c}36,64 \% \\
\text { (Chile Vamos-RN) }\end{array}$ & $\begin{array}{c}22,70 \% \\
\text { (La Fuerza de la Mayoria- } \\
\text { Independiente) }\end{array}$ & $13,94 \%$ & 4,17 \\
\hline \multicolumn{3}{|c|}{ Promedio MV-NEP (1989-2017) } & $18,43 \%$ & 2,94 \\
\hline
\end{tabular}

Fuente: elaboración propia a partir de Servel (s. f. b).

\footnotetext{
${ }^{9}$ Estimado mediante el número efectivo de partidos ([n54a12f01]) (Laakso y Taagepera, 1979), tomando el porcentaje de votos obtenidos para la elección presidencial.

${ }^{10}$ Entre paréntesis se registra la coalición electoral que obtuvo ese porcentaje de votos, seguido, guion mediante, del partido al que pertenece el candidato presidencial.
} 
La particularidad de las elecciones generales de 2017 radica en la menor concentración de votos obtenidos por los principales candidatos presidenciales, la volatilidad electoral presidencial y la fragmentación partidaria. Analizaremos estos indicadores de manera diacrónica para remarcar un punto central de este proceso electoral: el resultado de las elecciones generales de 2017 no es un hecho aislado y único, sino que se venía percibiendo en elecciones anteriores. En este sentido, se puede estar presenciando un punto de quiebre en la dinámica competitiva chilena que, sin embargo, aún no sabemos qué desenlace puede tomar.

En lo que respecta al total de votos obtenidos por los principales candidatos presidenciales, la gráfica 2 distingue entre la Concertación-Nueva Mayoría, Alianza-Chile Vamos, el tercer candidato y todos los restantes agrupados, desde el retorno a la democracia hasta las elecciones 2017.

Gráfica 2. Evolución votos totales elecciones presidenciales en Chile, 1989-2017.

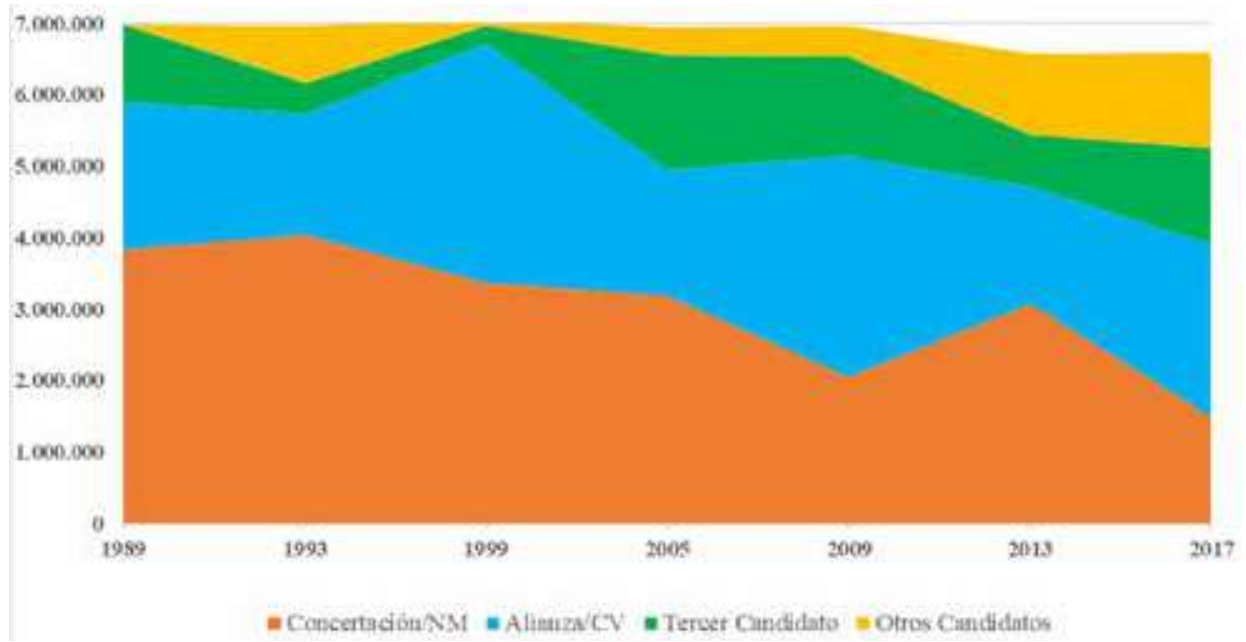

Fuente: elaboración propia a partir de Servel (s. f. b).

En primer lugar, se percibe cómo con el correr de los años la centroizquierda chilena comenzó a perder votos. En todo el periodo analizado solo registra un leve crecimiento entre 1989 y 1993, para posteriormente comenzar a decaer. Las elecciones de 2017, de hecho, muestran el peor resultado electoral de la coalición, incluso por debajo de la primera derrota sufrida en 2009. En segundo lugar, la centro-derecha muestra una tendencia menos estable en el periodo analizado, intercalando elecciones de 
crecimiento — casi duplicando sus votos entre 1993 y 1999, y entre 2005 y 2009-, con otros de pronunciada caída —entre 1999 y 2005-. De hecho, la victoria en las elecciones de 2017 no se logró con el mayor registro de votos totales en la primera vuelta, sino que alcanzaron un valor similar a la media de todo el periodo. En tercer lugar, en paralelo a la caída de la centroizquierda y la inestabilidad de la centro-derecha, se produjo un paulatino crecimiento en votos totales obtenidos por los candidatos presidenciales que lograron el tercer lugar en la contienda. Esto es particularmente notable en 2005 — cuando compitió Joaquín Lavín (UDI) por fuera de Alianza- pero especialmente relevante para este análisis en las elecciones 2009 y 2017. En estas dos oportunidades el tercer lugar fue alcanzado por otras coaliciones de centro-izquierda que plantearon un desafío al dominio de ese espacio para la Concertación-Nueva Mayoría. A partir de 2005 los chilenos que optaron por opciones diferentes a las coaliciones tradicionales alcanzaron alrededor de dos millones de personas —alrededor de un tercio de los electores-. De modo que la última década ha mostrado indicios de una dinámica competitiva que terminó de cristalizarse en las últimas elecciones.

En lo que respecta a la volatilidad electoral presidencial —índice de Pedersen-11 y a la fragmentación partidaria, la gráfica 3 sintetiza los dos indicadores. Sumamos también el cálculo de margen de victoria (MV) y la participación electoral para todo el periodo.

En lo que refiere a la participación electoral, es notable cómo el cambio del sistema de registro hacia uno de inclusión automática redujo la participación a la mitad del padrón electoral, confirmando la tendencia de Chile a una alta volatilidad en su tasa de participación electoral (Alcántara, Buquet y Tagina, 2018). Este es un punto no menor en un escenario de cuestionamiento y crisis de los partidos y coaliciones tradicionales en Chile (Olivares, 2018, agosto 7).

Por otro lado - y tal como se adelantó-, la evolución de la fragmentación partidaria para la competencia presidencial se produjo en paralelo con la ampliación del margen de victoria entre el primer y el segundo candidato. También se desprende de esto que el crecimiento de los terceros candidatos disparó el valor de NEPP por encima de los patrones regulares del sistema partidario chileno. El pico alcanzado en las elecciones presidenciales de 2017 mues-

\footnotetext{
${ }^{11}$ Indicador que estima la variación absoluta de los votos recibidos por cada partido en dos elecciones sucesivas (Pedersen, 1983).
} 
tra un cambio en la dinámica competitiva y un sistema más fragmentado a lo que se acostumbra en este país. Esto ubica a las últimas elecciones en valores similares a los de Brasil (Zucco, 2015) y Argentina (Malamud y De Luca, 2016).

Gráfica 3. Volatilidad, fragmentación

y participación electoral en Chile, 1989-2017.

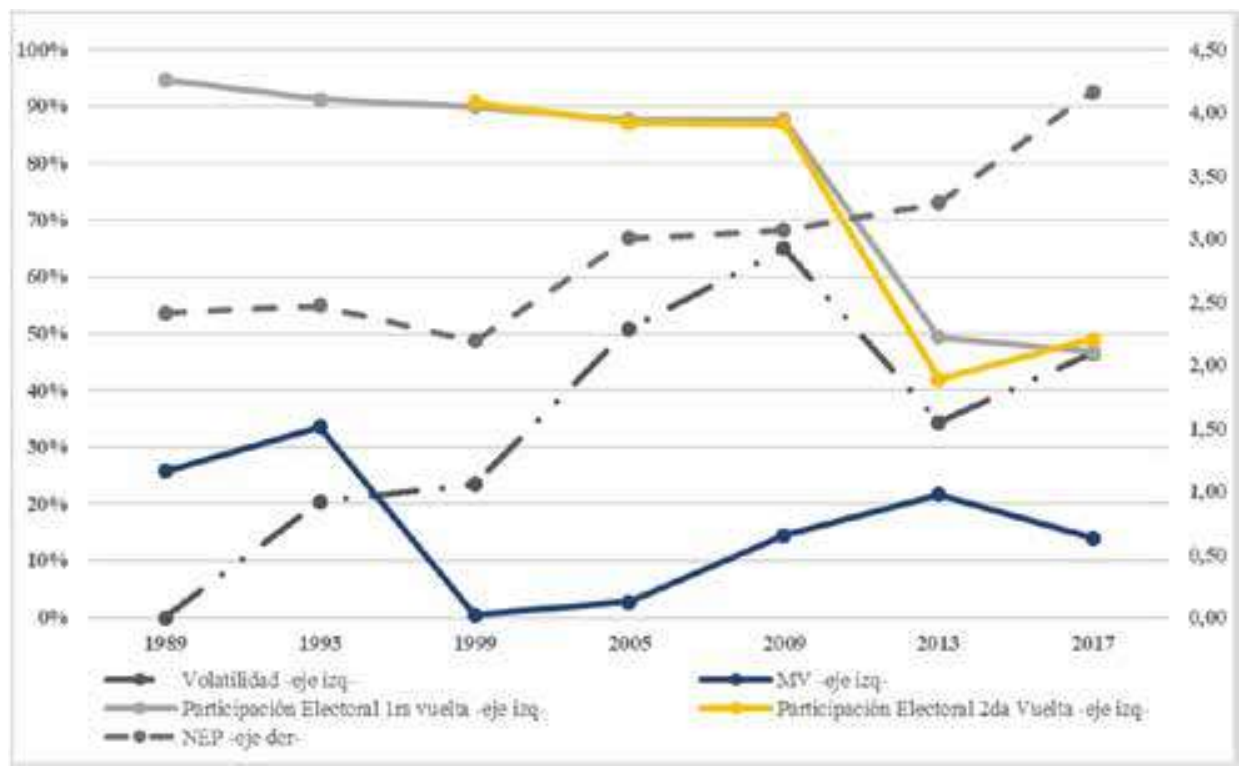

Fuente: elaboración propia a partir de Servel (s. f. b).

Este lento proceso de fragmentación partidaria se produjo en paralelo a una mayor volatilidad del comportamiento electoral para la disputa presidencial: el índice supera la barrera de 50\% a comienzos de 2000, hasta volver a descender y rondar $30-40 \%$ entre periodos consecutivos. Por lo anterior se puede considerar que la última década y media el sistema partidario chileno ha alternado ciclos de estabilidad y volatilidad marcados. En este sentido, el rara avis que representaba Chile junto a Uruguay en Latinoamérica pareciera estar culminando y acercando a Chile al grupo de los — levemente - impredecibles. Esto ratifica las dudas sobre la fortaleza de los partidos políticos en Chile y sus vínculos con el electorado (Luna y Altman, 2011; Luna y Rosenblatt, 2012, Siavelis, 2016).

De esta manera, el sistema partidario presidencial parece estar en el inicio de un proceso de cambio que, si bien se venía vislumbrando en las últimas dos elecciones y parece confirmarse con las celebradas en 2017, 
aún es pronto para conocer su formato y dinámicas futuras. Este punto no es menor, dado que el sistema electoral presidencial no se ha modificado sustancialmente desde 1989: siempre se han realizado elecciones bajo mayoría absoluta con doble vuelta. En este sentido, para comprender de manera más profunda los cambios a los que se hace mención, resulta necesario indagar sobre el sistema partidario legislativo.

\section{Un nuevo Congreso y un presidente conocido}

Los cambios sistémicos a los que se ha hecho mención también se manifestaron en las elecciones legislativas que se celebraron en conjunto con la primera vuelta presidencial. Sin dudas, se puede apreciar el efecto de la modificación del sistema electoral para el Congreso. Es altamente probable que estas alteraciones impactarán en la relación entre Poder Ejecutivo y Poder Legislativo durante la presidencia de Sebastián Piñera, dado que para su segundo mandato se enfrentará a una Asamblea mucho más fragmentada.

Antes de avanzar, una aclaración: la dinámica coalicional de competencia política (Siavelis, 2004; Buquet, 2015; Cruz, 2016) presenta un problema a la hora de analizar su sistema partidario. Dado que la oferta electoral se concentra en las coaliciones, esta situación puede nublar los vínculos de lealtad partidaria entre electores y candidatos (Luna y Altman, 2011). Para evitar este problema, se realizará el análisis de los indicadores pertinentes tanto para los partidos políticos como para las coaliciones.

En lo que respecta a la volatilidad del sistema de partidos legislativo, las elecciones de 2017 muestran un salto significativo respecto de las tendencias que caracterizaron a la democracia chilena desde el retorno a la democracia (véase gráfica 4). ${ }^{12}$

El índice de Pedersen muestra un paulatino crecimiento con el correr de los años, con un salto significativo a partir del cambio de reglas electorales. En lo que respecta al Senado, ha habido un comportamiento dispar al comparar partidos y coaliciones: más alto para los primeros, más bajo para los segundos. Para la Cámara de Diputados, en cambio, la diferencia entre ambas líneas no es muy grande, con la sola excepción del periodo 2001-1997 $-10 \%$ de diferencia-. Además de estos puntos, y en consonancia con lo dicho anteriormente, al acercarse a las elecciones de 2017 se encuentra que

${ }^{12}$ Los cálculos de volatilidad y NEP legislativo se realizaron sobre las bancas obtenidas por partidos políticos y coaliciones. 
todos los indicadores se estabilizan en torno a 30\%, valores que también son similares a la volatilidad electoral presidencial.

Gráfica 4. Volatilidad Presidencial y Legislativa (partidos y coaliciones). Chile, 1989-2017.

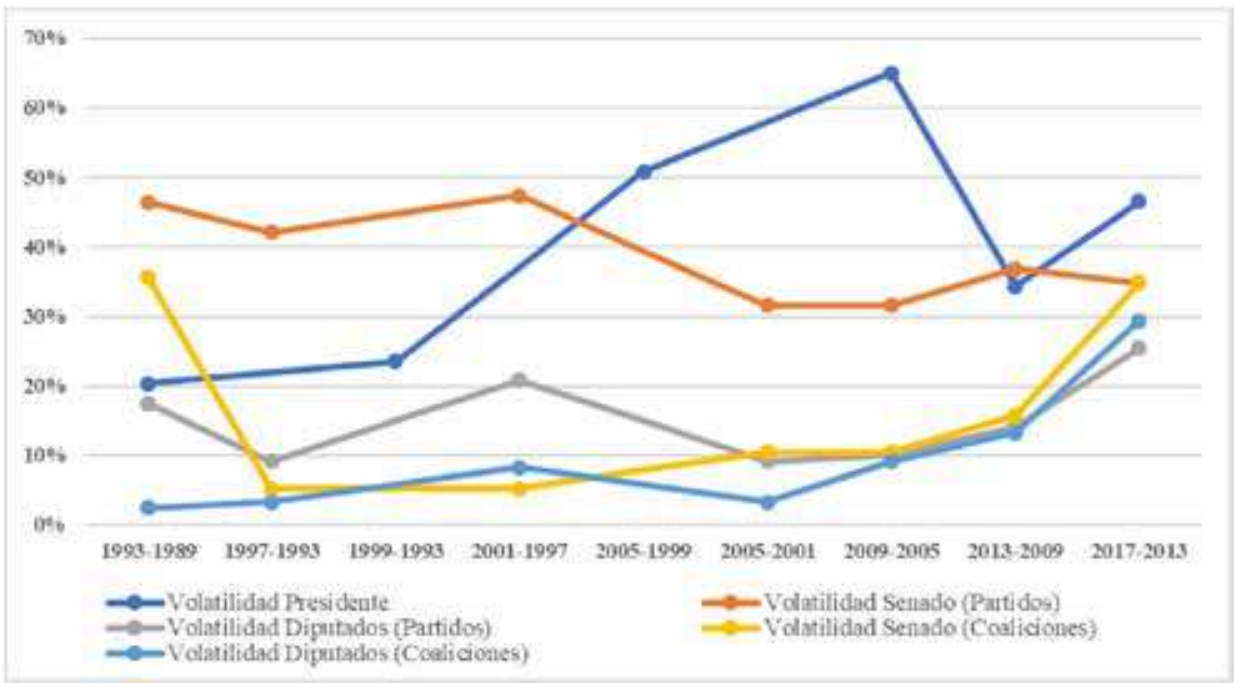

Fuente: elaboración propia a partir de Servel (s. f. b).

Este punto guarda cierta relación con lo planteado por Luna y Altman (2011), quienes encuentran que con el correr de los años cada vez menos electores chilenos se identifican con las dos coaliciones tradicionales. En consonancia con este trabajo, Rossana Castiglioni (2010) también anticipó el desencanto ciudadano con los actores tradicionales del sistema. El patrón de cambio que ya se venía vislumbrando en la competencia presidencial estaba siendo amortiguado en la competencia legislativa por el sistema binominal. Esto puede permitir considerar que el nuevo sistema electoral descomprimió los frenos que establecía el sistema anterior sobre el voto ciudadano. Dicho de otra manera: con a) nuevas reglas que permiten que nuevos partidos o coaliciones que crecen en votos crezcan también en bancas, y con b) un proceso de cambio en la identificación partidaria especialmente en los más jóvenes—, el sistema partidario chileno está en un punto de ebullición donde aún no queda claro la dinámica y la estructura que puede llegar a adquirir a futuro.

Esto también se percibe al indagar sobre el grado de fragmentación legislativa en ambas cámaras — medida a través del NEP con base en bancas 
obtenidas- La gráfica 5 compara la competencia presidencial con la distribución de bancas en el Congreso.

Gráfica 5. NEP presidencial y legislativo -partidos y coaliciones- en Chile, 1989-2017.

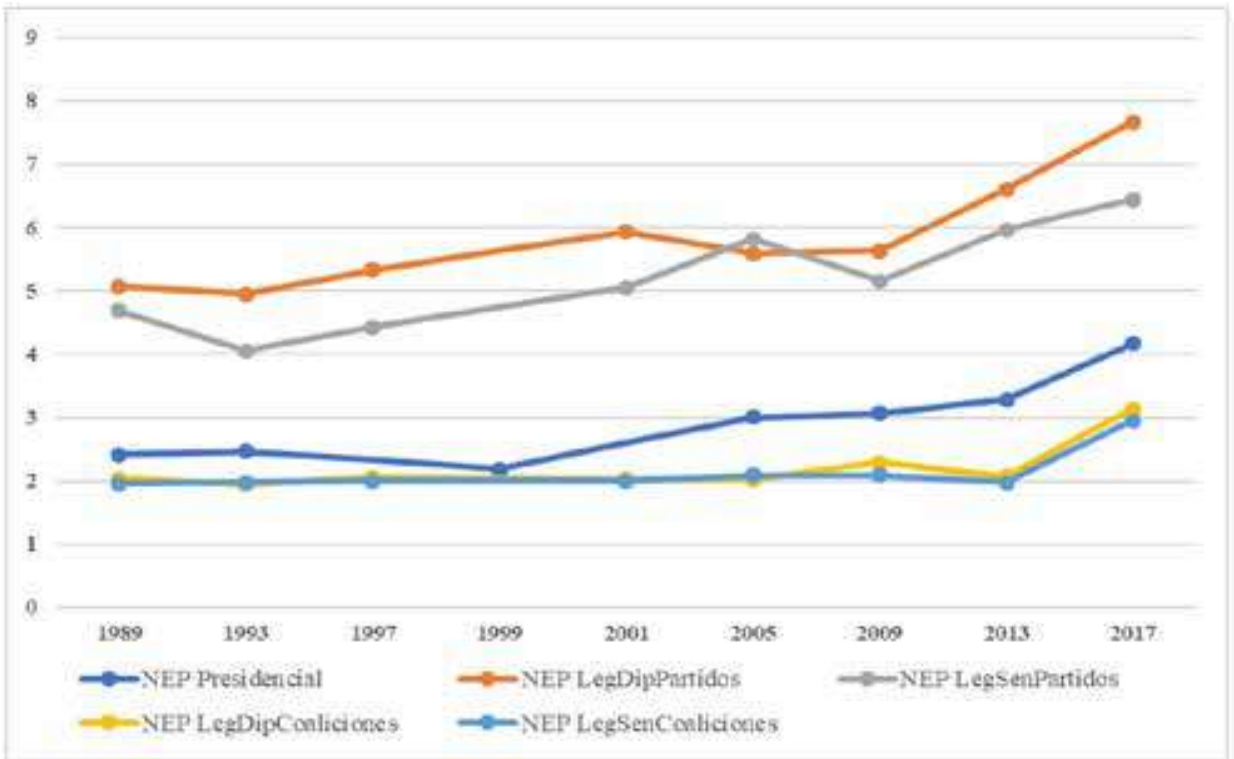

Fuente: elaboración propia a partir de Servel (s. f. b).

La fragmentación del Senado y de la Cámara de Diputados sigue una tendencia similar al de la volatilidad. Un periodo de concentración en dos grandes coaliciones - centro-izquierda y centro-derecha - hasta fines de la primera década de 2000, con un posterior incremento en la última elección de 2017. Si se toma en cuenta a los partidos políticos, el aumento de la fragmentación se produce en dos oleadas distintas: la primera a partir de 1997 hasta 2001; la segunda a partir de 2013, en el mismo momento en el cual la fragmentación del voto presidencial comienza a ser más marcada. Este punto refuerza la idea de la comprensión del sistema electoral binominal sobre el comportamiento electoral de los votantes chilenos. A pesar de este crecimiento, sin embargo, no se está frente a un Congreso dividido, como ha ocurrido en Brasil en las últimas décadas, sino más cercano al caso argentino (Malamud y De Luca, 2016) pero lejos del uruguayo (Buquet, 2015).

Las gráficas 6 y 7 complementan estos aspectos. Aquí se puede observar la evolución de la fragmentación partidaria con relación a las mayorías 
Crónica de un cambio anunciado. Las elecciones de 2017 en Chile frente al cambio...

legislativas de los gobiernos de turno. Esto es relevante en términos de la estrategia legislativa de Chile Vamos.

Gráfica 6. Fragmentación legislativa para Cámara de Diputados y Senado en Chile, 1989-2017.

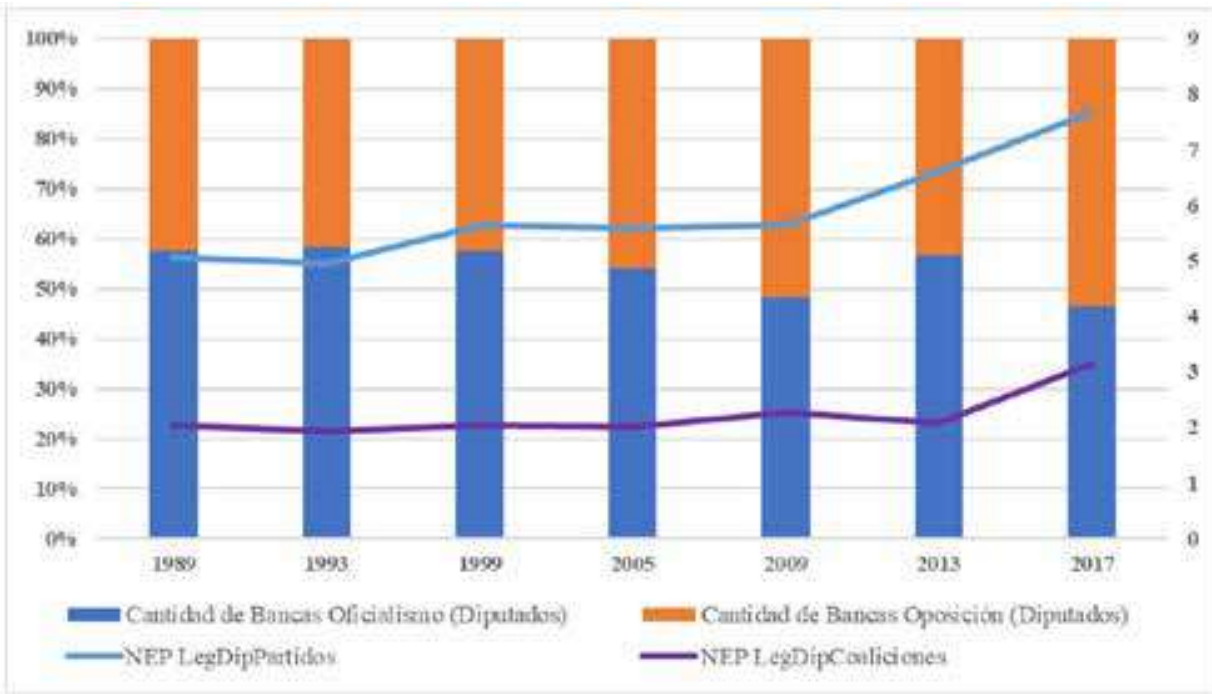

Fuente: elaboración propia a partir de Servel (s. f. b).

Gráfica 7. Mayorías de gobierno para Cámara de Diputados y Senado en Chile, 1989-2017.

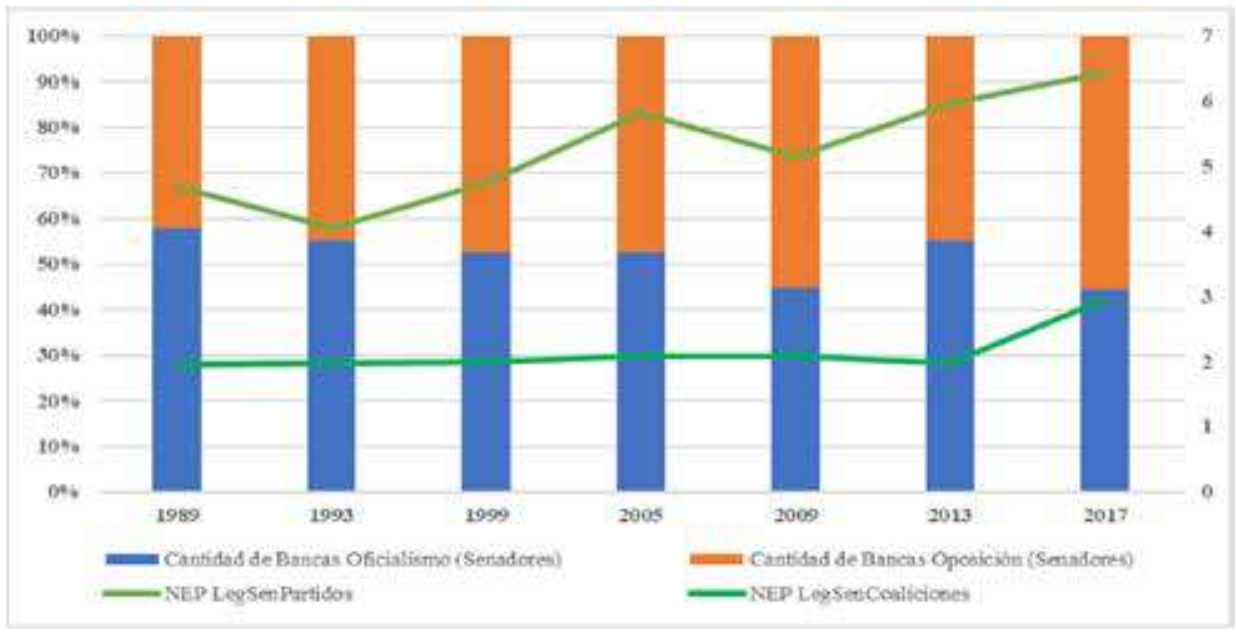

Fuente: elaboración propia a partir de Servel (s. f. b). 
El segundo mandato presidencial de Sebastián Piñera no será la excepción a la regla. Cuando su primera coalición electoral victoriosa, Alianza por Chile, llegó al gobierno en 2009 lo hizo con minoría en ambas Cámaras. Sin embargo, en esa oportunidad contaba con una oposición más abroquelada en una coalición de centro-izquierda dominada por los partidos de la Concertación. Esto se debe, en gran medida, a la mencionada dinámica coalicional característica del Congreso chileno desde 1989. En las primeras dos décadas los grandes bloques que han coordinado la negociación legislativa han sido la Concertación-Nueva Mayoría y Alianza-Chile Vamos. Durante los gobiernos concertacionistas la mayoría favorable se mantuvo con un alto nivel de disciplina legislativa (Toro, 2007).

El crecimiento de la fragmentación legislativa, sin embargo, abrió un periodo con interrogantes en torno a dos cuestiones centrales relacionadas con el vínculo Poder Ejecutivo-Poder Legislativo: primero, el ingreso del Frente Amplio como una nueva coalición de legisladores y senadores opositores al gobierno de turno - cuenta actualmente con 12,90\% de las bancas de diputados, pero tan solo 2,33\% de los senadores-, actores que no habían tenido mayor experiencia legislativa en periodos anteriores y que, adicionalmente, compiten por el espacio de centro-izquierda con los herederos de la Concertación: la Fuerza de la Mayoría. En este sentido, el doble juego de oposición frente a Chile Vamos y a la tradicional coalición progresista puede marcar ciertas pautas de competencia y conflicto al interior de la Cámara de Diputados.

Segundo, en torno al rol, carácter y posición que adoptará la Democracia Cristiana. Con posterioridad a su salida de la Concertación-Nueva Mayoría, la candidatura presidencial en solitario vino de la mano con listas propias de diputados y senadores para el Congreso. Actualmente cuenta con 13,95\% de los senadores y 9,03\% de los diputados: esto lo convierte en un actor atractivo tanto para el gobierno de turno como para sus exaliados. Estas bancas, sin duda, serán la clave para aprobar o bloquear la agenda legislativa de Piñera.

De modo que también se está frente a un proceso de cambio en el sistema partidario legislativo en Chile, no solo en lo que respecta a la alteración de los principales indicadores y sus tendencias respecto de los primeros veinte años desde la transición, sino también porque puede cambiar la dinámica colaborativa en el Congreso. Con más y nuevos actores se puede estar asistiendo a un periodo en el que los costos de negociación sean más 
altos. Tal como afirma Sergio Toro Maureira (2007), la persistencia del sistema binominal impactó positivamente en el grado de cohesión interna y de disciplina de los bloques legislativos en el Congreso chileno. Serán entonces los actores partidarios los que tendrán que acomodarse al nuevo esquema de incentivos al momento de actuar conjuntamente.

\section{Conclusiones}

Lo nuevo terminó por nacer. Los cambios que venían gestándose en el sistema partidario presidencial terminaron de materializarse en el sistema partidario legislativo con la modificación de las reglas de asignación de bancas y el aumento de las magnitudes de distrito. Si el sistema binominal funcionó como un contenedor del desencanto ciudadano con sus partidos y coaliciones tradicionales (Luna y Altman, 2011), las nuevas reglas tardarán un tiempo en acomodar el trayecto de la marea; aunque, si las previsiones de José Cabezas y Patricio Navia (2005) resultaran acertadas, solo implicará una moderada fragmentación que incorpore a las instituciones representativas muchas de las candidaturas que se sostuvieron a pesar del sistema binominal y de las dificultades para conseguir curules. Las elecciones generales de 2017 son el comienzo de una nueva estructura de la competencia política, en su dinámica y en su formato.

A partir de la comparación entre las últimas elecciones y las restantes celebradas desde el regreso a la democracia en 1989, se pueden sintetizar los hallazgos en tres etapas del sistema partidario: la primera entre 1989 y 2005, de estabilidad, baja fragmentación y alta institucionalización. La segunda entre 2005 y 2013, caracterizada por un desajuste entre el sistema partidario presidencial y el sistema partidario legislativo; en lo que respecta a la competencia

presidencial, se caracterizó por un aumento de la volatilidad electoral, un leve aumento de la fragmentación partidaria y un margen de victoria sostenido en torno al $10-15 \%$ entre los dos principales candidatos presidenciales; en cuanto a la dimensión legislativa, se mantuvo una baja fragmentación partidaria y una significativa injerencia de las dos coaliciones principales en la coordinación de los legisladores. La tercera, que comienza entre las elecciones 2013 y 2017, aún es un interrogante el desenlace que tendrá, ambos sistemas partidarios, el presidencial y el legislativo, comienzan a confluir nuevamente en lo que respecta a sus niveles de fragmentación y volatilidad electoral. Sin duda, el desempeño de los actores partidarios en este nuevo mandato de Sebastián Piñera marcará el rumbo que podrá tener la disputa política en la dinámica y el formato de la competencia política en Chile. 
Como suele ocurrir en la interacción entre actores e instituciones, estos acomodamientos llevan tiempo. El aprendizaje a partir de otros casos latinoamericanos con modificaciones significativas de las reglas electorales y de su impacto en los sistemas partidarios puede ayudar a traer calma. Argentina y Uruguay, por mencionar vecinos cercanos, pueden ser ejemplos interesantes para pensar la posible evolución de esta tercera etapa del sistema partidario chileno.

\section{Referencias bibliográficas}

1. Alcántara, Manuel, Buquet, Daniel y Tagina, María Laura. (2018). Introducción. En: Alcántara, Manuel, Buquet, Daniel y Tagina, María Laura (coords.). Elecciones y partidos en América Latina en el cambio de ciclo (pp. 7-20). Madrid: Centro de Investigaciones Sociológicas.

2. Altman, David y Luna, Juan Pablo. (2015). ¿Partidos hidropónicos en un sistema de partidos muy institucionalizado? El caso de Chile. En: Torcal, Mariano (coord.). Sistema de partidos en América Latina. Causas y consecuencias de su equilibrio inestable (pp. 203-219). Barcelona: Anthropos.

3. Altman, David y Toro Maureira, Sergio. (2016). Con el acelerador a fondo: la evaluación de las reformas a un año del gobierno de la Nueva Mayoría. En: CasasZamora, Kevin; Vidaurri, Marian; Muñoz-Pogossian, Betilde y Chanto, Raquel (eds.).

[254] Reformas políticas en América Latina: tendencias y casos (pp. 165-182). Washington, D. C.: SG/OEA.

4. Bardi, Luciano y Mair, Peter. (2008). The Parameters of Party Systems. Party Politics, 14 (2), pp. 147-166. https://doi.org/10.1177/1354068807085887

5. Buquet, Daniel. (2015). El cambio político en el Cono Sur: institucionalización partidaria y alternancia en Argentina, Chile y Uruguay. En: Torcal, Mariano (coord.). Sistema de partidos en América Latina. Causas y consecuencias de su equilibrio inestable (pp. 139-160). Barcelona: Anthropos.

6. Cabezas, José Miguel y Navia, Patricio. (2005). Efecto del sistema binominal en el número de candidatos y de partidos en elecciones legislativas en Chile, 1989-2001. Política. Revista de Ciencia Política, 45, pp. 29-51.

7. Castiglioni, Rossana. (2010). Chile y el giro electoral: «la vergüenza de haber sido y el dolor de ya no ser». Revista de Ciencia Política, 30 (2), pp. 231-248. https:// doi.org/10.4067/S0718-090X2010000200004

8. Cavarozzi, Marcelo y Casullo, Esperanza. (2002). Los partidos políticos en América Latina hoy: ¿Consolidación o crisis? En: Cavarozzi, Marcelo y Abal Medina, Juan Manuel (comps.). El asedio a la política. Los partidos latinoamericanos en la era neoliberal (pp. 9-30). Buenos Aires: Homo Sapiens.

9. Centro de Estudios Públicos. (s. f.). Julio-Agosto 2017. Estudio Nacional de Opinión Pública № 80. Recuperado de https://www.cepchile.cl/cep/site/ artic/20170831/asocfile/20170831165004/encuestacep_jul_ago2017.pdf 
Crónica de un cambio anunciado. Las elecciones de 2017 en Chile frente al cambio...

10. Cooperativa.cl. (2017, abril 9). Partido Socialista ya definió: Guillier es su candidato. Recuperado de https://www.cooperativa.cl/noticias/ pais/politica/presidenciales/partido-socialista-ya-definio-guillier-es-sucandidato/2017-04-09/170109.html

11. Cooperativa.cl. (2017, noviembre 8). Alejandro Guillier presentó su programa de Gobierno. Recuperado de https://www.cooperativa.cl/noticias/ pais/politica/presidenciales/alejandro-guillier-presento-su-programa-degobierno/2017-11-08/011107.html

12. Coppedge, Michael. (1998). The Dynamic Diversity of Latin American Party Systems. Party Politics, 4 (4), pp. 547-568. https://doi. org/10.1177/1354068898004004007

13. Cruz, Facundo. (2016). Volatilidad y competitividad electoral en América Latina. Un estudio exploratorio de seis sistemas partidarios. Revista Colección, 26, pp. 163-211.

14. De la Paz, Patricio. (2017, diciembre). José Antonio Kast: «Muchos del mundo homosexual van a votar por mí». T13. Recuperado de http://www.t13.cl/noticia/ politica/semanal/jose-antonio-kast-muchos-del-mundo-homosexual-van-votar-mi

15. Diario Uchile. (2017, octubre 21). «El programa de muchos»: La propuesta con que Beatriz Sánchez espera Ilegar a La Moneda. Recuperado de https://radio.uchile. cl/2017/10/21/el-programa-de-muchos-la-propuesta-con-la-que-beatriz-sanchezespera-Ilegar-a-la-moneda/

16. Došek, Tomas. (2016). Cambios y continuidades en el sistema de partidos de Chile (1989-2015): entre la estabilidad y el desencanto. En: Freidenberg, Falvia (ed.). Los sistemas de partidos en América Latina. 1978-2015. Tomo 2. Cono Sur y países andinos (pp. 135-192). México, D. F.: UNAL.

17. El Desconcierto. (2017, septiembre 27). Plebiscito del Frente Amplio: 16.793 personas participaron del inédito proceso programático. Recuperado de http:// www.eldesconcierto.cl/2017/09/27/plebiscito-del-frente-amplio-16-793-personasparticiparon-del-inedito-proceso-programatico/

18. Emol. (2016, mayo 23). Integrantes de Revolución Democrática renuncian al Ministerio de Educación. Recuperado de https://www.emol.com/noticias/ Nacional/2016/05/23/804156/Integrantes-de-Revolucion-Democratica-renuncian-alMinisterio-de-Educacion.html

19. Fortín Mapocho. (2017, octubre 23). El regionalismo de Alejandro Guillier: Entregó plan de descentralización de Chile. Recuperado de https://www. fortinmapocho.cl/2017/10/23/el-regionalismo-de-alejandro-guillier-entrego-su-plande-descentralizacion-del-pais/

20. Goić Boroevic, Carolina. (2017). Volvamos a confiar. Ejes programáticos. Democracia Cristiana. Servel. Recuperado de https://www.servel.cl/wp-content/ uploads/2017/09/Programa_Carolina_Goic_Boroevic.pdf 
21. Guillier, Alejandro. (2017). Programa de gobierno Alejandro Guillier Álvarez. Servel. Recuperado de https://www.servel.cl/wp-content/uploads/2017/09/Programa_ Alejandro_Guillier_Alvarez.pdf

22. Guzmán, Nicolás. (2017, octubre 31). Goic presenta programa de gobierno con énfasis en salud y crecimiento. La Tercera. Recuperado de https://www.latercera. com/noticia/goic-presenta-programa-gobierno-enfasis-salud-crecimiento/

23. Laakso, Markku y Taagepera, Rein. (1979). "Effective" Number of Parties: A Measure with Application to West Europe. Comparative Political Studies, 12 (1), pp. 3-27. https://doi.org/10.1177/001041407901200101

24. López Varas, Miguel y Baeza Freer, Jaime. (2013). Las elecciones chilenas de 2009-2010: ¿se derechizó el país? En: Alcántara, Manuel y Tagina, María Laura (coords.). Elecciones y política en América Latina 2009-2011 (pp. 301-328). México, D. F.: Instituto Nacional Electoral.

25. Luna, Juan Pablo y David Altman. (2011). Uprooted but Stable: Chilean Parties and the Concept of Party System Institutionalization. Latin American Politics and Society, 53 (2), pp. 1-28. https://doi.org/10.1111/j.1548-2456.2011.00115.x

26. Luna, Juan Pablo y Rosenblatt, Fernando. (2012). ¿Notas para una autopsia? Los partidos políticos en el Chile actual. En: Díaz, Javier y Sierra, Lucas (eds.). Democracia con partidos. Informe para la reforma de los partidos políticos en Chile (pp. 115-267). Santiago de Chile: CEP y Cieplan.

27. Malamud, Andrés y De Luca, Miguel. (2016). ¿Todo sigue igual que ayer?

[256] Continuidad y ruptura en el sistema de partidos argentino (1983-2015). Los sistemas de partidos en América Latina (1978-2015). Tomo 2. Cono Sur y países andinos (pp. 27-68). México, D. F.: UNAM.

28. Montes, Rocío. (2017, noviembre 13). El presidenciable chileno que reivindica a Pinochet. El País. Recuperado de https://elpais.com/internacional/2017/11/13/ america/1510527795_264102.html

29. Morgan, Jana y Meléndez, Carlos. (2016). Parties Under Stress: Using a Linkage Decay Framework to Analyze the Chilenean Party System. Politics in Latin America, 8 (3), pp. 25-59.

30. NTN24. (2017, diciembre 12). Las cinco propuestas fuertes con las que Sebastián Piñera y Alejandro Guillier buscan ganar la Presidencia de Chile. Recuperado de http://archivo.ntn24.com/noticia/cinco-propuestas-fuertes-con-las-que-sebastianpinera-y-alejandro-guillier-buscan-llegar-a-la-160217

31. Olivares, Alejandro. (2018, agosto 7). Alerta a los partidos. La Tercera. Recuperado de https://www.latercera.com/la-tercera-pm/noticia/alerta-lospartidos/272533/

32. Opinión Global. (2017, julio 17). Programa de gobierno de José Antonio Kast. Recuperado de http://www.opinionglobal.cl/programa-de-gobierno-de-jose-antoniokast-resumen/ 
Crónica de un cambio anunciado. Las elecciones de 2017 en Chile frente al cambio...

33. Pedersen, Mogens. (1983). Changing Patterns of Electoral Volatility in European Party Systems, 1948-1977: Explorations in Explanation. En: Daalder, Hans y Mair, Peter (eds.). Western European Party Systems: Continuity and Change (pp. 29-66). Londres: Sage.

34. Piñera, Sebastián. (2017). Tiempos mejores. Servel. Recuperado de https:// www.servel.cl/wp-content/uploads/2017/09/Programa_Sebastian_Pinera_Echenique. pdf

35. Scully, Timothy. (1995). Building Democratic Institutions: Party Systems in Latin America. Stanford: Stanford University.

36. Siavelis, Peter. (2000). Continuidad y cambio en el sistema partidista chileno: sobre los efectos de transformación de una reforma electoral. Revista de Ciencia Política, 20 (2), pp. 82-102.

37. Siavelis, Peter. (2004). Sistema electoral, desintegración de coaliciones y democracia en Chile: ¿El fin de la Concertación? Revista de Ciencia Política, 24 (1), pp. 58-80. https://doi.org/10.4067/S0718-090X2004000100003

38. Siavelis, Peter. (2016). Crisis of Representation in Chile? The Institutional Connection. Politics in Latin America, 8 (3), pp. 61-93.

39. Seira, Iván; Santamarina, Sofía; Falak, Agustina; Casas, Federico y Farias Guizzo, Julia. (2017). \#EleccionesChile: distintas reglas, ¿mismos actores? Asuntos del Sur. Recuperado de https://www.asuntosdelsur.org/opinion/eleccioneschile-distintasreglas-mismos-actores

40. Servicio Electoral de Chile (Servel). (2015, agosto 18). Nuevo sistema electoral chileno: Método D'Hondt. Recuperado de https://www.servel.cl/nuevo-sistemaelectoral-chileno-metodo-dhont-2/

41. Servicio Electoral de Chile (Servel). (2017, julio 2). Último boletín público y solemne de resultados preliminares de las elecciones primarias 2017 para presidente de la República. Recuperado de https://www.servel.cl/wp-content/uploads/2017/07/1_1_ Primarias Presidente.pdf

42. Servicio Electoral de Chile (Servel). (s. f. a). Voto exterior. Recuperado de https://www.servel.cl/voto-exterior/

43. Servicio Electoral de Chile (Servel). (s. f. b). Resultados históricos. Recuperado de https://historico.servel.cl/

44. Sistema de Crédito Estudios Superiores (Ingresa). (s. f.). Características del crédito. Recuperado de https://portal.ingresa.cl/preguntas-frecuentes/\#pregunta_533

45. Sputnik. (2017, octubre 19). Candidato chileno Enríquez-Ominami: «Mi campaña es la más transparente». Recuperado de https://mundo.sputniknews.com/ americalatina/201710191073327428-santiago-elecciones-entrevista/

46. Torcal, Mariano y Mainwaring, Scott. (2003). The Political Recrafting of Social Bases of Party Competition: Chile, 1973-95. British Journal of Political Science, 33 (1), pp. 55-84. https://doi.org/10.1017/S0007123403000036 
47. Toro Maureira, Sergio. (2007). Conducta legislativa ante las iniciativas del Ejecutivo: unidad de los bloques políticos en Chile. Revista de Ciencia Política, 27 (1), pp. 23-41. https://doi.org/10.4067/S0718-090X2007000200002

48. Toro Maureira, Sergio y Valenzuela, Macarena. (2018). Chile 2017: ambiciones, estrategias y expectativas en el estreno de las nuevas reglas electorales. Revista de Ciencia Política, 38 (2), pp. 207-232. https://doi.org/10.4067/s0718090x2018000200207

49. Zucco Jr, Cesar. (2015). Estabilidad sin raíces: La institucionalización del sistema de partidos brasileño. En: Torcal, Mariano (coord.). Sistemas de partidos en América Latina. Causas y consecuencias de su equilibrio inestable (pp. 78-107): Santa Fe: Anthropos-UNL. 